\title{
On Optimal Link Creation for Facilitation of Consensus in Social Networks
}

\author{
Makan Fardad, Fu Lin, and Mihailo R. Jovanović
}

\begin{abstract}
We consider the problem of reaching consensus in a social network of agents described by the DeGroot model. We develop a measure for the efficiency with which consensus is reached, where the measure quantifies the transient behavior of public opinion around the consensus value. We then propose an optimization problem that maximizes consensusreaching efficiency via the creation of new social links, subject to a total link-creation budget. We employ the alternating direction method of multipliers, an algorithm well-suited to large optimization problems, to find the optimal location and weights of the new links. We demonstrate the utility of our results through an example, where we observe that for a social network described by a regular graph the addition of new links leads to an augmented graph that resembles a small-world network characterized by sparse long-range links.
\end{abstract}

Index Terms - Alternating direction method of multipliers, consensus, DeGroot model, opinion dynamics, optimization, small-world networks, social networks, sparsity, stochastic matrices.

\section{INTRODUCTION}

Problems in social networks, such as social influence, community detection, opinion formation, consensus, cascades, and viral marketing, have been the topic of active research across different scientific communities; see [1]-[13] and references therein. Most of these works share the common theme of analyzing a certain network property or behavior over an a priori given topology of social interactions. In contrast, in this work we search over all possible augmentations of the existing network so as to optimize its performance in achieving a common goal.

We consider a social network of single-integrator agents whose beliefs evolve in time according to the classic DeGroot model [14]. In this model every agent updates its opinion by taking a weighted average of those it socially interacts with. Networks governed by such dynamics are known to reach consensus in steady-state. However, steady-state analysis does not capture the system's transient characteristics, such as short-time amplification and oscillations around the consensus value. Thus, in this paper we begin by developing a measure for the efficiency with which consensus is reached. Our measure is based

Financial support from the National Science Foundation under awards CNS-1329885, CMMI-0927509, and CMMI-0927720 is gratefully acknowledged.

M. Fardad is with the Department of Electrical Engineering and Computer Science, Syracuse University, Syracuse, NY 13244. F. Lin is with the Mathematics and Computer Science Division, Argonne National Laboratory, Argonne, IL 60439. M. R. Jovanović is with the Department of Electrical and Computer Engineering, University of Minnesota, Minneapolis, MN 55455. E-mails: makan@syr.edu, fulin@mcs.anl.gov, mihailo@umn.edu. on the deviation of individual opinions from a common opinion, summed over all agents and over all time. We then formulate an optimization problem which, given a certain budget for creating new social links over the entire network, attempts to maximize the efficiency of reaching consensus by choosing both the best locations and the best weights for new social links. This optimization problem is complicated by the fact that, in order to incorporate the beliefs of new individuals, an agent has to make room for new social links by reconfiguring its weighted averaging scheme.

We use the alternating direction method of multipliers (ADMM) to solve the optimization problem, and apply our results to a social network described by a regular graph with nearest neighbor interactions. We find that the optimal new social interactions are in the form of sparse long-range links, resembling the characteristics of a small-world network [15]

\section{Quantifying Consensus EFFICIENCY}

Consider a social network composed of $n$ agents whose beliefs evolve according to

$$
x(k+1)=T x(k) .
$$

Here, $x$ is a column $n$-vector composed of nonnegative values that represent the beliefs of the agents with regards to a particular social issue, and $T$ is a (right) stochastic matrix that satisfies

$$
T \mathbb{1}=\mathbb{1}, \quad T_{i j} \geq 0, i, j=1, \ldots, n,
$$

where $\mathbb{1}$ is the column vector of all ones. The matrix $T$ is not necessarily symmetric. We assume that all diagonal entries of $T$ are positive and that the graph described by $T$ is strongly connected; these conditions guarantee that the network will reach consensus in steady-state [8], [14], and that all eigenvalues of $T$ except for the one at $\lambda=1$ belong to the open unit disk. The belief evolution scheme described by (1)-(2) is widely used in the social networks literature and is often referred to as the DeGroot model.

In a network described by (1)-(2), at every time step each agent updates his/her beliefs by taking a weighted average of the beliefs of those agents he/she socially interacts with. This justifies why every row of $T$ is composed of nonnegative entries that sum to one. It is not difficult to see that if such a system is initialized at an $x(0)$ whose entries all belong to the interval $[0,1]$, then the entries of $x(k)$ remain within this interval for all $k \geq 0$. Furthermore, it can be shown that under our assumptions on $T$, the network reaches consensus 
as $k$ grows,

$$
\lim _{k \rightarrow \infty} x(k)=\chi \mathbb{1}
$$

where the consensus value $\chi$ is determined by a weighted average of the entries of $x(0)$ with weights that depend on the matrix $T$. Of course, this result concerns only the steady-state behavior of the social network. In what follows, we propose a new measure of consensus that accounts for transient behavior around $\chi$ and quantifies the efficiency with which the consensus opinion is reached, regardless of the value of $\chi$.

Let $Q$ be a positive semidefinite matrix that satisfies $Q \mathbb{1}=$ o, i.e.,

$$
\mathbb{1}^{T} Q \mathbb{1}=0, \quad \zeta^{T} Q \zeta \geq 0 \text { for all } \zeta \neq 0 \text { s.t. } \zeta^{T} \mathbb{1}=0 .
$$

The properties of $Q$ imply that $x(k)^{T} Q x(k)$ quantifies the deviation of the entries of $x(k)$ from a uniform value. Let $e_{i}$ denote the $i$ th standard basis vector in $\mathbb{R}^{n}$, and let

$$
x(0)=e_{i} .
$$

This can be interpreted as a social network in which all agents have zero initial belief except for the $i$ th agent, whose belief is equal to one. From (1) it follows that the propagation of this initial belief through society is described by

$$
x(k)=T^{k} e_{i},
$$

and

$$
x(k)^{T} Q x(k)=e_{i}^{T} T^{k T} Q T^{k} e_{i}
$$

measures the deviation from consensus at time $k$.

Proceeding formally, we define

$$
J=\sum_{i=1}^{n}\left(e_{i}^{T} Q e_{i}+e_{i}^{T} T^{T} Q T e_{i}+e_{i}^{T} T^{2 T} Q T^{2} e_{i}+\cdots\right)
$$

as a measure of consensus-reaching efficiency. We have

$$
\begin{aligned}
J= & \operatorname{trace}\left(Q\left(\sum_{i} e_{i} e_{i}^{T}\right)+T^{T} Q T\left(\sum_{i} e_{i} e_{i}^{T}\right)\right. \\
& \left.\quad+T^{2 T} Q T^{2}\left(\sum_{i} e_{i} e_{i}^{T}\right)+\cdots\right) \\
= & \operatorname{trace}\left(Q+T^{T} Q T+T^{2 T} Q T^{2}+\cdots\right) \\
= & \operatorname{trace}(P)
\end{aligned}
$$

where $P$ is a positive semidefinite matrix that satisfies the algebraic Lyapunov equation

$$
P=T^{T} P T+Q \text {. }
$$

Indeed, the smaller $J$ is the more efficient the convergence of public opinion to the consensus value.

We emphasize again that compared to other consensus performance measures in the literature, e.g., distance from average consensus in [8], the measure defined by $J$ is independent of the actual consensus value $\chi$ and only depends on the cumulative transient behavior around $\chi$.

In defining $J$ in the preceding development, we temporarily ignored issues of convergence. However, since $T$ satisfies $T \mathbb{1}=\mathbb{1}$ and therefore has an eigenvalue at $\lambda=1$, we have to ensure that the infinite series in the definition of $J$ converges. The key observation here is that when $Q$ satisfies $Q \mathbb{1}=0$ it effectively makes the mode at $\lambda=1$ unobservable, thus allowing the infinite sum in the definition of $J$ to converge. Lemma 1 below makes this notion precise. Furthermore, Lemma 1 describes how to modify the Lyapunov equation (3) so that eigenvalue at $\lambda=1$ can be moved to within the open unit disk in the complex plane. Our result is quite general in that it does not require the matrix $T$ to be symmetric, or its corresponding graph to be undirected, as opposed to a similar result in [16], [17] which utilizes the symmetry of $T$. We define the notation $\mathbb{1}^{\perp}=\left\{x \in \mathbb{R}^{n} \mid \mathbb{1}^{T} x=0\right\}$.

Lemma 1: Let the matrices $\mathcal{A}$ and $\mathcal{Q}$ satisfy $\mathcal{A} \mathbb{1}=\mathbb{1}$ and $\mathcal{Q} \mathbb{1}=0, \mathcal{Q}^{T}=\mathcal{Q}$. Suppose that $\mathcal{A}$ is diagonalizable and has spectrum in the open unit disk when restricted to the subspace $\mathbb{1}^{\perp}$, and that $\mathcal{Q}$ is a positive semidefinite matrix when restricted to the subspace $\mathbb{1}^{\perp}$. Then among all positive semidefinite solutions of the Lyapunov equation

$$
\mathcal{P}=\mathcal{A}^{T} \mathcal{P} \mathcal{A}+\mathcal{Q},
$$

the one with the minimum trace satisfies

$$
\mathcal{P} \mathbb{1}=0 .
$$

Furthermore, this minimum-trace solution also satisfies

$$
\mathcal{P}=\left(\mathcal{A}-\mathbb{1}^{T} / n\right)^{T} \mathcal{P}\left(\mathcal{A}-\mathbb{1}^{T} / n\right)+\mathcal{Q},
$$

where $\mathcal{A}-1 \mathbb{1}^{T} / n$ has spectrum in the open unit disk.

Proof: An outline of the proof is as follows. We use the properties $\mathcal{A} \mathbb{1}=\mathbb{1}$ and $\mathcal{Q} \mathbb{1}=0$ to show that any matrix $\mathcal{P}$ that satisfies $\mathcal{P}=\mathcal{A}^{T} \mathcal{P} \mathcal{A}+\mathcal{Q}$ necessarily has $\mathbb{1}$ as an eigenvector, $\mathcal{P} \mathbb{1}=\omega \mathbb{1}$, and that the corresponding eigenvalue $\omega$ can take any nonnegative real value (independently of the other eigenvalues). Since the trace of the positive semidefinite matrix $\mathcal{P}$ is equal to the sum of its (nonnegative) eigenvalues, then $\operatorname{trace}(\mathcal{P})$ is minimized if $\omega=0$, and thus $\mathcal{P} \mathbb{1}=0$. This implies that if one solves the Lyapunov equation $\mathcal{P}=\left(\mathcal{A}-\beta \mathbb{1}^{T} / n\right)^{T} \mathcal{P}\left(\mathcal{A}-\beta \mathbb{1}^{T} / n\right)+\mathcal{Q}$ subject to the condition $\mathcal{P} \mathbb{1}=0$ then the solution is independent of $\beta$. Therefore $\beta$ can be chosen to shift the eigenvalue of $\mathcal{A}$ at 1 to inside the open unit disk; in particular, $\beta=1$ shifts this eigenvalue to 0 . The details of the proof are omitted due to space limitations and will be reported elsewhere.

Using this result, in what follows we replace the Lyapunov equation in (3) with

$$
P=\left(T-\mathbb{1}^{T} / n\right)^{T} P\left(T-\mathbb{1}^{T} / n\right)+Q .
$$

\section{CReation of New Social Links}

In this section we consider the following problem: Suppose that we are allotted a total amount of weight $\varrho$ that we can use to create new social links, i.e., if the $i$ th agent creates new social links whose weights sum to $\sigma_{i}$, then $\sum_{i=1}^{n} \sigma_{i} \leq \varrho$. Obviously $\varrho$ has to be a small number for this to be an interesting problem; if $\varrho$ is very large, then the result of creating new links may be a dense graph in which every agent is connected to all other agents. 
Let us recall that in the framework considered in this work, each agent updates its belief by taking a weighted average over the beliefs of those it socially interacts with. Thus, for an agent to create new links between itself and other agents, it has to 'make room' in its weighted averaging scheme by 'scaling down' the weight it puts on existing links.

The evolution of beliefs in the new augmented social network is described by the dynamics [18], [19]

$$
x(k+1)=(S T+U) x(k)
$$

where

$$
\begin{gathered}
S=I-\operatorname{diag}\{\sigma\}, \\
U \mathbb{1}=\sigma, \quad U_{i j} \geq 0, \\
\sigma=\left[\begin{array}{lll}
\sigma_{1} & \cdots & \sigma_{n}
\end{array}\right]^{T}, \\
0 \leq \sigma_{i} \leq 1 \text { for } i=1, \ldots, n,
\end{gathered}
$$

$\sigma_{i}=$ total weight agent $i$ allocates to its new social links.

The matrix $U$ describes the weight and distribution of the new social links; the entries in the $i$ th row of $U$ sum to $\sigma_{i}$. The matrix $S$ scales down the weights of the existing links to make room for new ones; the $i$ th diagonal element of $S$ is equal to $1-\sigma_{i}$. As expected, $S T+U$ is a stochastic matrix, as its entries are all positive and its rows sum to one,

$$
(S T+U) \mathbb{1}=S T \mathbb{1}+U \mathbb{1}=S \mathbb{1}+\sigma=\mathbb{1}-\sigma+\sigma=\mathbb{1} .
$$

We now formulate an optimization problem with the purpose of finding the vector of weights $\sigma$ and the distribution of the weights $U$ within the network such that consensus-reaching efficiency is maximally raised, subject to the constraint $\mathbb{1}^{T} \sigma \leq \varrho$ on the sum of weights,

$$
\begin{array}{ll}
\operatorname{minimize} & \operatorname{trace}(P) \\
\text { subject to } & P=\left(S T+U-\mathbb{1}^{T} / n\right)^{T} P . \\
& \cdot\left(S T+U-\mathbb{1}^{T} / n\right)+Q, \quad P \mathbb{1}=0 \\
& S=I-\operatorname{diag}\{\sigma\} \\
& U \mathbb{1}=\sigma, \quad U \geq 0 \\
& \mathbb{1}^{T} \sigma \leq \varrho, \quad 0 \leq \sigma \leq \mathbb{1} .
\end{array}
$$

The Lyapunov equation in the constraints uses Lemma 1 with $\mathcal{A}:=S T+U$ and $\mathcal{Q}:=Q$, and is a substitute for the equation $P=(S T+U)^{T} P(S T+U)+Q$; see the discussion preceding Lemmal for a justification of this substitution.

We note that in this formulation the number of links is not explicitly penalized. Therefore, the possibilities in the solution of (4) range from 'all agents making many new social links with small weights' (which would correspond to a dense $U$ with many small entries) to 'some agents making a few (perhaps long-range) links with large weights' (which would correspond to both a sparse $\sigma$ and a sparse $U$ with a few large entries). In the examples section, we will solve (4) for some simple problems and demonstrate that solutions indeed display sparse properties.

\section{Solving Social Link Creation Problem}

In this section, we examine the structure of the social link creation problem (4). While it is a nonconvex problem, (4) can be formulated as a minimization problem with a nonlinear objective function over a set of linear constraints. This observation motivates the use of the alternating direction method of multipliers (ADMM) [20], which decomposes (4) into a sequence of simpler problems. Specifically, ADMM alternates between solving an unconstrained nonlinear program and solving a quadratic program with linear constraints.

We begin by rewriting problem (4) in the form

$$
\begin{array}{ll}
\text { minimize } & J(\sigma, U):=\operatorname{trace}(P) \\
\text { subject to } & P=W^{T} P W+Q, \quad P \mathbb{1}=0 \\
& W=S T+U-\mathbb{1}^{T} / n \\
& S=I-\operatorname{diag}\{\sigma\} \\
& U \mathbb{1}=\sigma, \quad U \geq 0 \\
& \mathbb{1}^{T} \sigma \leq \varrho, \quad 0 \leq \sigma \leq \mathbb{1},
\end{array}
$$

where optimization variables are the $n$-vector $\sigma$ and the $n \times n$ matrices $P, W, S, U$. Here, $\sigma, U$ are design variables of interest and $P, W, S$ are auxiliary variables for computing the objective value $J$. Note that (5) is a nonconvex optimization problem because the Lyapunov equation imposes a nonlinear equality constraint on variables $W$ and $P$.

To examine its structure, we next rewrite problem (5) as

$$
\begin{array}{ll}
\operatorname{minimize} & J(\sigma, U) \\
\text { subject to } & U \mathbb{1}=\sigma, \quad U \geq 0 \\
& \mathbb{1}^{T} \sigma \leq \varrho, \quad 0 \leq \sigma \leq \mathbb{1},
\end{array}
$$

where the consensus-reaching efficiency $J(\sigma, U)$ is computed as follows. For given $(\sigma, U)$, we form $S$ and $W$,

$$
\begin{aligned}
S & =I-\operatorname{diag}\{\sigma\} \\
W & =S T+U-\mathbb{1}^{T} / n,
\end{aligned}
$$

solve the Lyapunov equation

$$
P=W^{T} P W+Q,
$$

and take the trace of $P$ to obtain $J$. Note that the auxiliary variables $P, W, S$ are eliminated. Thus, $J$ is a nonlinear function of $(\sigma, U)$ and the constraints in (6) are linear in $(\sigma, U)$. This structure is exploited next using ADMM.

We introduce the indicator function of the linear constraint set

$$
\phi(\sigma, U)= \begin{cases}0 & (\sigma, U) \in \mathcal{C} \\ \infty & \text { otherwise }\end{cases}
$$

where

$$
\mathcal{C}:=\left\{(\sigma, U) \mid U \mathbb{1}=\sigma, U \geq 0, \mathbb{1}^{T} \sigma \leq \varrho, 0 \leq \sigma \leq \mathbb{1}\right\} .
$$

Then (5) can be put into the following form

$$
\text { minimize } J(\sigma, U)+\phi(\sigma, U),
$$


or equivalently,

$$
\begin{array}{ll}
\operatorname{minimize} & J(\sigma, U)+\phi(z, Z) \\
\text { subject to } & \sigma=z, \quad U=Z .
\end{array}
$$

The reformulation (7) aims at separating the nonlinear objective function $J$ from the set of linear constraints $\mathcal{C}$. This is done by introducing the auxiliary variables $(z, Z)$ such that $J$ and $\phi$ are defined over two sets of optimization variables. As we show below, the ADMM algorithm exploits this separable structure and solves (7) through a sequence of simpler problems. Specifically, it solves the constrained nonlinear program (7) with a sequence of unconstrained nonlinear programs and linearly constrained quadratic programs.

To this end, we form the augmented Lagrangian function of the constrained problem (7)

$$
\begin{array}{r}
\mathcal{L}_{\rho}(\sigma, U, z, Z, \psi, \Psi)=J(\sigma, U)+\phi(z, Z)+\psi^{T}(\sigma-z) \\
+\operatorname{trace}\left(\Psi^{T}(U-Z)\right)+\frac{\rho}{2}\left(\|\sigma-z\|_{2}^{2}+\|U-Z\|_{F}^{2}\right)
\end{array}
$$

where $\psi \in \mathbb{R}^{n}, \Psi \in \mathbb{R}^{n \times n}$ are Lagrange multipliers and $\rho$ is a positive scalar. The ADMM algorithm solves (7) using the following sequence of iterations

$$
\begin{aligned}
(\sigma, U)^{k+1} & :=\underset{\sigma, U}{\arg \min } \mathcal{L}_{\rho}\left(\sigma, U, z^{k}, Z^{k}, \psi^{k}, \Psi^{k}\right) \\
(z, Z)^{k+1} & :=\underset{z, Z}{\arg \min } \mathcal{L}_{\rho}\left(\sigma^{k+1}, U^{k+1}, z, Z, \psi^{k}, \Psi^{k}\right) \\
\psi^{k+1} & :=\psi^{k}+\rho\left(\sigma^{k+1}-z^{k+1}\right) \\
\Psi^{k+1} & :=\Psi^{k}+\rho\left(U^{k+1}-Z^{k+1}\right)
\end{aligned}
$$

until the primal and dual residues are sufficiently small,

$$
\begin{array}{cl}
\text { primal residues: } & \left\{\begin{array}{l}
\left\|\sigma^{k}-z^{k}\right\|_{2} \leq \epsilon_{1} \\
\left\|U^{k}-Z^{k}\right\|_{F} \leq \epsilon_{2}
\end{array}\right. \\
\text { dual residues: } & \left\{\begin{array}{l}
\left\|\sigma^{k}-\sigma^{k+1}\right\|_{2} \leq \epsilon_{3} \\
\left\|z^{k}-z^{k+1}\right\|_{2} \leq \epsilon_{4} \\
\left\|U^{k}-U^{k+1}\right\|_{F} \leq \epsilon_{5} \\
\left\|Z^{k}-Z^{k+1}\right\|_{F} \leq \epsilon_{6}
\end{array}\right.
\end{array}
$$

where $\epsilon_{i}$ are determined by problem data; see [20, Section 3.3] for details.

Note that the $(\sigma, U)$-minimization step (8a) is an unconstrained nonlinear program, which can be solved using descent methods (e.g., gradient or quasi-Newton iterations [21]). We provide expressions for the gradient direction

$$
\begin{aligned}
\nabla_{\sigma} \mathcal{L}_{\rho}(\sigma, U) & =-2 \operatorname{diag}\left\{T M W^{T} P\right\}+\rho\left(\sigma-\pi^{k}\right) \\
\nabla_{U} \mathcal{L}_{\rho}(\sigma, U) & =2 P W M+\rho\left(U-\Pi^{k}\right),
\end{aligned}
$$

where $\pi^{k}=z^{k}-\psi^{k} / \rho$ and $\Pi^{k}=Z^{k}-\Psi^{k} / \rho$. Here,

$$
W=(I-\operatorname{diag}\{\sigma\}) T+U-\mathbb{1}^{T} / n,
$$

$P$ and $M$ are solutions of the Lyapunov equations

$$
\begin{aligned}
P & =W^{T} P W+Q \\
M & =W M W^{T}+I .
\end{aligned}
$$

For given $(\sigma, U)$, we form $W$ in (10), compute $P$ and $M$ by solving the Lyapunov equations in (11), and evaluate the gradient direction in (9).

Using a gradient method, the computational complexity of problem (8a) is of order $n^{3}$, where $n$ is the number of agents in a social network. This is because the evaluation of the gradient direction (9) requires solutions of Lyapunov equations (11), which require $O\left(n^{3}\right)$ operations.

We next turn to the $(z, Z)$-minimization step (8b). This step amounts to minimizing a quadratic objective function subject to linear constraints

$$
\begin{array}{ll}
\operatorname{minimize} & \frac{\rho}{2}\left(\left\|z-v^{k}\right\|^{2}+\left\|Z-V^{k}\right\|_{F}^{2}\right) \\
\text { subject to } & Z \mathbb{1}=z, \quad Z \geq 0, \quad \mathbb{1}^{T} z \leq \varrho, \quad 0 \leq z \leq \mathbb{1},
\end{array}
$$

where $v^{k}=\sigma^{k+1}+\psi^{k} / \rho$ and $V^{k}=U^{k+1}+\Psi^{k} / \rho$. Eliminating $z$ yields

$$
\begin{array}{ll}
\operatorname{minimize} & \frac{\rho}{2}\left(\left\|Z \mathbb{1}-v^{k}\right\|^{2}+\left\|Z-V^{k}\right\|_{F}^{2}\right) \\
\text { subject to } & Z \geq 0, \quad \mathbb{1}^{T} Z \mathbb{1} \leq \varrho, \quad 0 \leq Z \mathbb{1} \leq \mathbb{1} .
\end{array}
$$

Since this is a convex quadratic program, its unique solution can be computed efficiently by solving the resulting KKT conditions (see e.g., [21, Section 6.4]).

\section{An EXAMPLE}

We consider a social network with $n=25$ agents on a circle; see Fig. 2. The weight that agent $i$ puts on its own belief is determined by $T_{i i}=0.5+0.01 r_{i}$, where $r_{i}$ is a Gaussian random variable with zero mean and unit variance. Each agent distributes the remaining weight $1-T_{i i}$ equally on its neighbors and therefore $T$ is right stochastic.

We solve (5) for $\varrho \in[0.01,0.1]$ with 10 uniform grid points. The consensus measure $J$ decreases as $\varrho$ increases; see Fig. 1a. This is expected, since a bigger $\varrho$ implies a bigger feasible set for $(\sigma, U)$, and hence a smaller optimal objective value. On the other hand, it is observed that the belief adjustment matrix $U$ is an approximately sparse matrix. Specifically, it has a few relatively large entries (on the order of $10^{-3}$ ) and many small entries (on the order of $10^{-9}$ ). In particular, the number of entries greater than $10^{-3}$ in $U$ increases with $\varrho$; see Fig. $1 \mathrm{~b}$.

This approximately sparse solution $U$ is perhaps rather surprising in view of the fact that no sparsity-promoting penalty function (such as the $\ell_{1}$ norm) is introduced in (5). However, a closer look at (5) reveals that the constraints on $(\sigma, U)$ play the sparsity-promoting role. It is instructive to examine the $(z, Z)$-minimization step in the ADMM iterations. The resulting quadratic program (12) amounts to a projection on the intersection of the positive orthant $Z \geq 0$ with the polyhedron determined by $\left\{\mathbb{1}^{T} Z \mathbb{1} \leq \varrho\right.$, $0 \leq Z \mathbb{1} \leq \mathbb{1}\}$. Such a projection tends to be on the vertices, yielding a sparse solution. Also note that $\varrho$ controls the size of the polyhedron and hence the level of sparsity of the solution. 


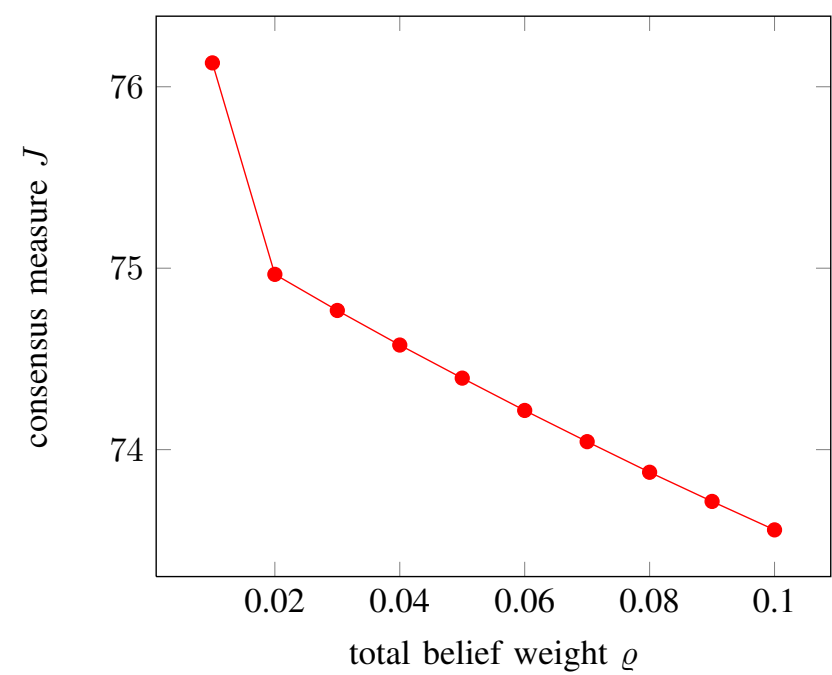

(a)

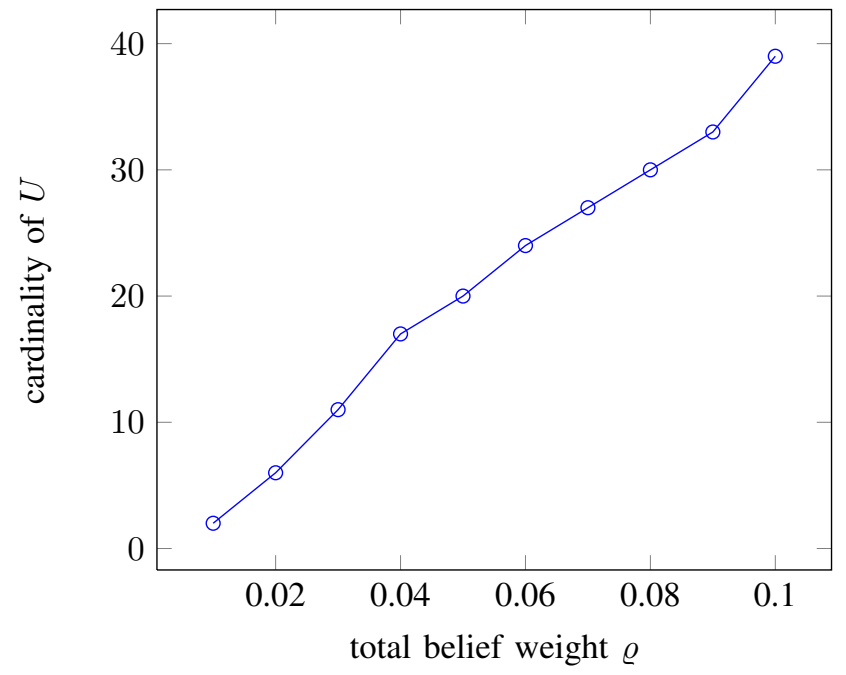

(b)

Fig. 1: The consensus measure $J$ and the number of social links as a function of total belief weight $\varrho$.

We next examine the structure of the new (augmented) social networks. As shown in Fig. 2, the new social links are formed among agents that are far from each other. These long-range interactions facilitate consensus, as they significantly shorten the average distance between any pair of nodes in the social network. This is one of the main characteristics of the small-world phenomenon that has been observed in a variety of networks (e.g, see [15]).

\section{CONCLUDING REMARKS}

We consider the efficiency of reaching consensus in a social network governed by the DeGroot model. We propose a performance measure that quantifies the transient behavior of opinion dynamics. Based on this new measure, we consider the optimal creation of new social links subject to budget constraints. We develop an ADMM algorithm that exploits the separable structure of the optimization problem.

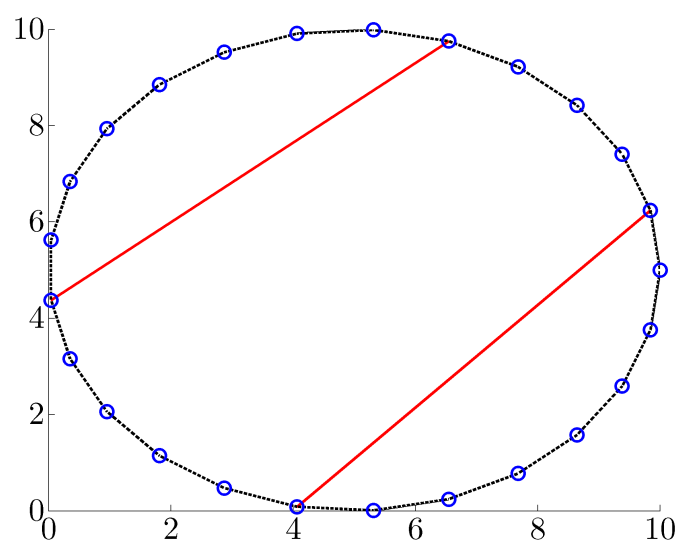

(a) $\varrho=0.01$

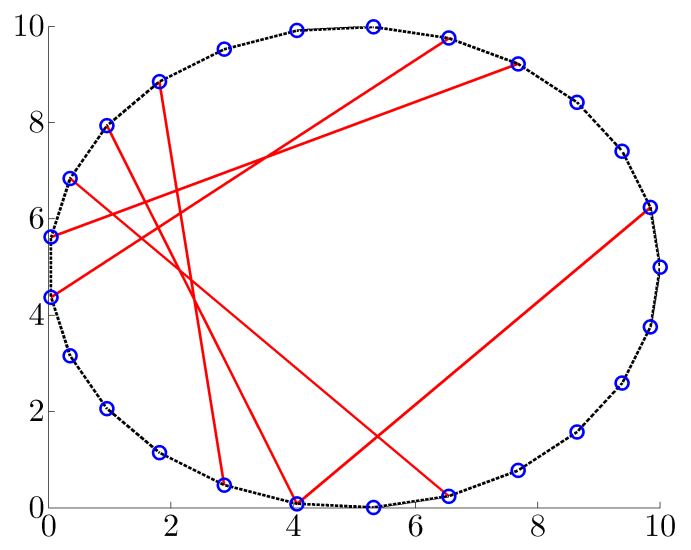

(b) $\varrho=0.02$

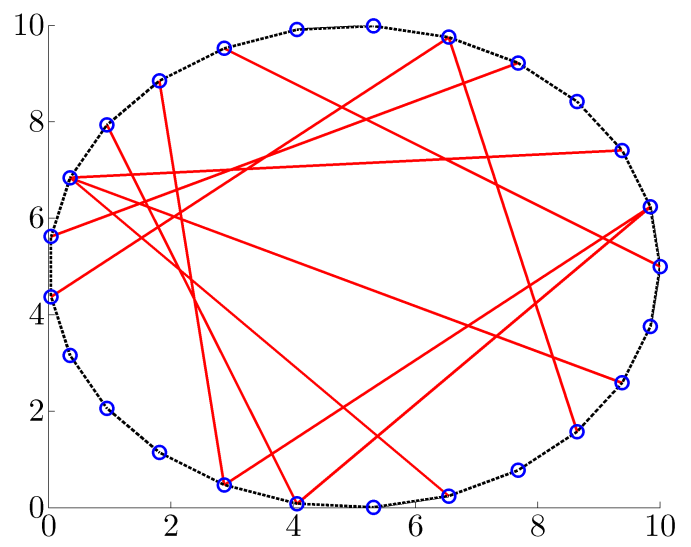

(c) $\varrho=0.03$

Fig. 2: A social network with $n=25$ agents on a circle with local interactions between neighbors indicated by (black) dash lines and long-range interactions indicated by (red) solid lines. 
We observe in examples that new social links are created among non-local agents. Indeed, it is possible to employ a perturbation framework to demonstrate that the set of optimal weak social links is both sparse and long-range, when the network is allocated a small amount of resources with which to make new links. This result will be the topic of future research.

\section{REFERENCES}

[1] P. M. DeMarzo, D. Vayanos, and J. Zwiebel, "Persuasion bias, social influence, and unidimensional opinions," The Quarterly Journal of Economics, vol. 118, no. 3, pp. 909-968, 2003.

[2] M. E. J. Newman, "The structure and function of complex networks," SIAM Review, vol. 45, no. 2, pp. 167-256, 2003.

[3] S. P. Borgatti and M. G. Everett, "A graph-theoretic perspective on centrality," Social Networks, vol. 28, pp. 466-484, 2006.

[4] B. Golub and M. Jackson, "Naive learning social networks and the wisdom of crowds," American Economic Journal: Microeconomics, vol. 2, no. 1, pp. 112-149, 2010.

[5] D. Kempe, J. Kleinberg, and E. Tardos, "Maximizing the spread of influence through a social network," in Proceedings of KDD, 2003.

[6] H. Amini, M. Draief, and M. Lelarge, "Marketing in a random network," in Network Control and Optimization (Lecture Notes in Computer Science Volume 5425). Springer, 2009, pp. 17-25.

[7] V. Blondel1, J.-L. Guillaume, R. Lambiotte, and E. Lefebvre, "Fast unfolding of communities in large networks," Journal of Statistical Mechanics: Theory and Experiment, vol. 10, p. P10008, 2008.

[8] F. Fagnani and S. Zampieri, "Randomized consensus algorithms over large scale networks," IEEE Journal on Selected Areas in Communications, vol. 26, pp. 634-649, 2008.

[9] R. Carli, F. Fagnani, A. Speranzon, and S. Zampieri, "Communication constraints in the average consensus problem," Automatica, vol. 44, p. 671684, 2008.

[10] L. Blume, D. Easley, J. Kleinberg, R. Kleinberg, and E. Tardos, "Which networks are least susceptible to cascading failures?" in Proceedings of the 52nd IEEE Symposium on Foundations of Computer Science, 2011.

[11] F. Chierichetti, J. Kleinberg, and A. Panconesi, "How to schedule a cascade in an arbitrary graph," in Proceedings of the 13th ACM Conference on Electronic Commerce, 2012.

[12] G. Como and F. Fagnani, "Scaling limits for continuous opinion dynamics systems," Annals of Applied Probability, vol. 21, p. 15371567 , 2011.

[13] P. Frasca, C. Ravazzi, R. Tempo, and H. Ishii, "Gossips and prejudices: Ergodic randomized dynamics in social networks," 2013, arXiv: 1304.2268.

[14] M. H. DeGroot, "Reaching a consensus," Journal of the American Statistical Association, vol. 69, pp. 118-121, 1974.

[15] D. J. Watts and S. H. Strogatz, "Collective dynamics of 'small-world' networks," Nature, vol. 393, pp. 440-442, 1998.

[16] M. Fardad, F. Lin, and M. R. Jovanović, "On the optimal synchronization of oscillator networks via sparse interconnection graphs," in Proceedings of the 2012 American Control Conference, 2012, pp. 4777-4782.

[17] M. Fardad, F. Lin, and M. R. Jovanović, "Design of optimal sparse interconnection graphs for synchronization of oscillator networks," to appear in IEEE Transactions on Automatic Control, 2014, doi:10.1109/TAC.2014.2301577.

[18] M. Fardad, X. Zhang, F. Lin, and M. R. Jovanović, "On the optimal dissemination of information in social networks," in Proceedings of the 51st IEEE Conference on Decision and Control, 2012, pp. 25392544.

[19] M. Fardad, F. Lin, X. Zhang, and M. R. Jovanović, "On new characterizations of social influence in social networks," in Proceedings of the 2013 American Control Conference, 2013, pp. 4784-4789.

[20] S. Boyd, N. Parikh, E. Chu, B. Peleato, and J. Eckstein, "Distributed optimization and statistical learning via the alternating direction method of multipliers," Foundations and Trends in Machine Learning, vol. 3, no. 1, pp. 1-122, 2011.

[21] J. Nocedal and S. J. Wright, Numerical Optimization. Springer, 2006. 